\title{
Enterotoxin production by coagulase-negative staphylococci in restaurant workers from Kuwait City may be a potential cause of food poisoning
}

\author{
E. E. UDO, M. A. AL-BUSTAN*, L. E. JACOB and T. D. CHUGH \\ Departments of Microbiology and ${ }^{*}$ Community Medicine, Faculty of Medicine, Kuwait University, Kuwait
}

\begin{abstract}
Staphylococcus aureus and coagulase-negative staphylococci (CNS) were isolated from the hands of food handlers in $\mathbf{5 0}$ restaurants in Kuwait City and studied for the production of staphylococcal enterotoxins, toxic shock syndrome toxin-1, slime and resistance to antimicrobial agents. One or a combination of staphylococcal enterotoxins $\mathrm{A}, \mathrm{B}$ or $\mathrm{C}$ were produced by $6 \%$ of the isolates, with the majority producing enterotoxin B. Toxic shock syndrome toxin-1 was detected in $c$. $7 \%$ of the isolates; $47 \%$ produced slime. In all, $21 \%$ of the isolates were resistant to tetracycline and $11.2 \%$ were resistant to propamidine isethionate and mercuric chloride. There was no correlation between slime and toxin production or between slime production and antibiotic resistance. The detection of enterotoxigenic CNS on food handlers suggests that such strains may contribute to food poisoning if food is contaminated by them and held in conditions that allow their growth and elaboration of the enterotoxins. It is recommended that enterotoxigenic CNS should not be ignored when investigating suspected cases of staphylococcal food poisoning.
\end{abstract}

\section{Introduction}

Staphylococcus aureus and coagulase-negative staphylococci (CNS) inhabit the human skin and mucous membranes where they exist mostly as commensal flora [1-3]. However, some of them also cause different types of infections in man and animals. $S$. aureus is an important cause of food poisoning through the elaboration of one or more of six serologically related, heat-stable enterotoxins designated staphylococcal enterotoxins (SE) A-E [4-8]. Ingestion of these preformed toxins in contaminated food leads to symptoms of food poisoning [6-8].

Some CNS species are recognised human pathogens and can cause serious opportunist infections, especially in immunocompromised patients and premature babies [9-13], with the patients' skin flora serving as a major source of the infecting micro-organisms. They also elaborate a number of important toxins and haemolysins implicated in serious disease conditions. For example, some CNS produce staphylococcal toxic shock syndrome toxin-1 (TSST-1) which causes toxic shock syndrome [14-16], $\delta$-toxins associated with

Received 1 Sept. 1998; revised version accepted 4 Jan. 1999. Corresponding author: Dr E. E. Udo (e-mail: EDET@hsc. kuniv.edu.kw). necrotising enterocolitis in premature infants [17] and $\delta$-haemolysin associated with chronic orofacial muscle pain [18]. Furthermore, staphylococcal enterotoxins (SE) have been detected in CNS isolates from food [16-21], goat's milk [22] and other animal products $[23,24]$. Despite these findings, studies that have investigated the sources of staphylococcal food poisoning have focused almost exclusively on the carriage of enterotoxigenic $S$. aureus [7, 8, 25-28], and rarely on enterotoxin-producing CNS [16, 25], in food handlers. As the human nares and fingers are the important sources of $S$. aureus contaminating food in restaurants $[25,26]$ and because CNS inhabit the human skin and mucous membranes, they can also contaminate food from the hands of food handlers if proper care is not taken when preparing and serving food. Therefore, enterotoxigenic CNS strains may contribute to staphylococcal food poisoning, as any enterotoxigenic staphylococcal strain can be involved in food poisoning [29]. In a study of nasal carriage of $S$. aureus by restaurant workers in Kuwait City, it was found that $26 \%$ of them carried $S$. aureus in their nares and $86 \%$ of these $S$. aureus isolates produced either TSST-1 or SE. The nasal swabs were not screened for CNS carriage or their toxins.

In the present study, nasal and hand swabs from workers in different restaurants in Kuwait City were 
cultured for the presence of $S$. aureus and CNS and the isolates were examined for the production of staphylococcal enterotoxins, TSST- 1 and slime and for resistance to antimicrobial agents.

\section{Materials and methods}

\section{Sample collection}

A total of 500 nasal and hand swabs was obtained from 250 food handlers in 50 restaurants. The personnel included those who handle food in some way such as cutting, washing, cooking and serving. Sterile swabs (Atom Medical, Sussex) were moistened in sterile nutrient broth. One swab was used to swab areas in between fingers and the wrist area of the hand and another swab was used to swab the nose. The swabs were used to inoculate mannitol salt agar (MSA) and blood agar plates in duplicate and the plates were incubated at $35^{\circ} \mathrm{C}$. Cultures on blood agar were examined after $24 \mathrm{~h}$ and those on MSA were examined after incubation for $48 \mathrm{~h}$. Representatives of each colony type were subcultured on blood agar and incubated at $35^{\circ} \mathrm{C}$ for $24 \mathrm{~h}$ for identification.

\section{Identification of staphylococcal strains}

The isolates were identified on the basis of cultural characteristics, Gram's stain reaction and the results of catalase and tube coagulase tests. Tube coagulase negative, catalase positive, gram-positive coccal isolates were identified further with biochemical tests (API 20 Staph identification system; bioMérieux SA, Marcy l'Etoile, France). Pure cultures of the isolates were preserved in skimmed milk at $-20^{\circ} \mathrm{C}$. They were subcultured in brain heart infusion broth and incubated at $35^{\circ} \mathrm{C}$. Working cultures were maintained on agar slopes at $4^{\circ} \mathrm{C}$. $S$. aureus strain ATCC 25923 and $S$. epidermidis strain ATCC 12228 were used as control strains.

\section{Susceptibility to antimicrobial agents}

Susceptibility to antimicrobial agents was tested by the disk diffusion method as described previously [30], with Mueller Hinton Agar (Unipath, Basingstoke). The disks were impregnated with the following antibacterial agents $(\mu \mathrm{g})$ : methicillin (5), penicillin $(10)$, gentamicin (10), kanamycin (30), neomycin (30), streptomycin (30), erythromycin (15), clindamycin (2), chloramphenicol (30), tetracycline (10), minocycline (30), trimethoprim (2.5), fusidic acid (10), rifampicin (5), ciprofloxacin (5), mupirocin (200), teicoplanin (30), vancomycin (30), cadmium acetate (50), mercuric chloride (100), propamidine isethionate $(50)$ or ethidium bromide $(60)$. The plates were incubated at $35^{\circ} \mathrm{C}$ for $24 \mathrm{~h}$. Propamidine isethionate was a gift from Rhône-Poulenc Rorer, UK.

\section{Detection of staphylococcal toxins}

SE and TSST-1 production were detected by the reverse passive latex agglutination kits SET-RPLA and TSTRPLA (Unipath), respectively. Both tests were performed by following the manufacturer's instructions. Known positive and negative controls accompanied each test sample.

\section{Slime production}

Slime production was detected by the qualitative method as described by Jones et al. [31]. Pure cultures of the organisms were grown in $5 \mathrm{ml}$ of trypticase soy broth supplemented with glucose $10 \% \mathrm{w} / \mathrm{v}$ and incubated at $35^{\circ} \mathrm{C}$ for $18 \mathrm{~h}$ in glass test tubes in a slanting position. The broth culture was then decanted and the slime layer adherent to the glass surface was stained with $5 \mathrm{ml}$ of safranin $(0.25 \mathrm{~g}$ in $100 \mathrm{ml}$ of ethanol $95 \%$ ). The tubes were allowed to dry before the results were read. When no stained slime or only a trace of film was observed, the isolate was reported as slimenegative. Organisms forming an extensive film of slime were designated slime-positive.

\section{Results}

\section{Distribution of staphylococcal isolates}

A total of 174 isolates of staphylococcal species consisting of $32 \mathrm{~S}$. aureus and $142 \mathrm{CNS}$ was isolated from the hands of healthy food handlers in different restaurants in Kuwait City. In contrast $157 \mathrm{~S}$. aureus $(92.3 \%)$ and only 13 CNS (7.6\%) isolates were cultured from the nares of the workers. The 13 nasal CNS isolates consisted of six $S$. hominis $(3.8 \%)$, four $S$. epidermidis $(2.5 \%)$ and three $S$. warneri $(1.9 \%)$. The nasal $S$. aureus isolates were not studied further as part of this report.

CNS obtained from the workers' hands consisted of 41 S. hominis $(28.8 \%), 36 \mathrm{~S}$. warneri $(25.3 \%), 17 \mathrm{~S}$. saprophyticus (11.9\%), 17 S. xylosus (11.9\%), 12 $S$. schleiferi $(8.4 \%)$, six $S$. epidermidis $(4.2 \%)$, six $S$. haemolyticus $(4.2 \%)$, two $S$. cohnii $(1.4 \%)$ and one each of $S$. capitis, $S$. intermedius and $S$. lentus. Two isolates could not be identified with the API-Staph identification system.

\section{Toxin production}

Twenty-four isolates (13.8\%), consisting of four $S$. aureus $(12.5 \%)$ and $20 \mathrm{CNS}(14.1 \%)$, produced either SE or TSST-1. Thirteen isolates - two S. aureus, 10 CNS $(5.6 \%)$ from the hands and one nasal $S$. hominis-produced TSST-1. Fifteen isolates - four $S$. aureus and $11 \mathrm{CNS}$ - produced different SEs. Eleven of the 15 SE-isolates produced SEB; only three isolates produced SEA. Three isolates produced both SEA and SEB. None of them produced SED or SEE. Four isolates, two $S$. aureus and one each of 
S. saprophyticus and $S$. warneri, produced both SE and TSST (Table 1).

\section{Antimicrobial resistance}

Resistance to different antimicrobial agents was detected in all CNS species. However, only a small number were resistant to antibiotics. All were susceptible to vancomycin, teicoplanin, rifampicin, gentamicin, streptomycin and ciprofloxacin and 47 (33.1\%) were susceptible to all antimicrobial agents tested. Thirty $(21.1 \%)$ were resistant to tetracycline, 10 to methicillin, five to erythromycin, six to trimethoprim and nine to chloramphenicol; and three isolates (one $S$. saprophyticus and two $S$. xylosus) expressed high-level resistance to mupirocin.

Sixteen isolates $(11.2 \%)$ were resistant to the nucleic acid binding compound, propamidine isethionate, and to mercuric chloride. Sixty-two isolates were resistant to cadmium. Twenty-six isolates $(18.3 \%)$, were resistant to three or more of the antimicrobial agents and were classified as multi-resistant. Five of the nasal CNS isolates were resistant to tetracycline and six were resistant to cadmium. Of the $32 \mathrm{~S}$. aureus strains isolated from the workers' hands, 14 and 10 of them were resistant to penicillin $G$ and tetracycline, respectively, five were resistant to chloramphenicol and four were resistant to mercuric chloride and propamidine isethionate.

\section{Slime production}

Slime production was demonstrated in $67(46.1 \%)$ of the CNS isolates. These consisted of $16(39.0 \%)$ of 41 $S$. hominis, $15(41.6 \%)$ of $36 \mathrm{~S}$. warneri, 14 (82.3\%) of $17 \mathrm{~S}$. xylosus, six of $17 \mathrm{~S}$. saprophyticus, five of 12 $S$. schleiferi, four of six $S$. haemolyticus, three of six $S$. epidermidis, both $S$. cohnii and one of the two unspeciated isolates. None of the $S$. capitis, $S$. intermedius and $S$. lentus isolates produced slime. There was no correlation between slime and toxin production, as the majority of the isolates that produced slime did not produce toxin and, as summarised in Table 1, many of the isolates that produced toxins did not produce slime, although some slimeproducing isolates also produced different toxins. Also, there was no correlation between slime production and antibiotic resistance or between toxin production and antibiotic resistance.

\section{Discussion}

This study investigated $S$. aureus and CNS isolates from the hands of food handlers working in restaurants in Kuwait City, for SE and slime production and for resistance to antimicrobial agents. The study revealed that $\mathrm{CNS}$ were more prevalent than $S$. aureus as hand colonisers among these restaurant workers. In contrast, CNS constituted only $7 \%$ of staphylococci from the noses of the same workers. In Spain, CNS constituted

Table 1. Characteristics of strains producing TSST and SE

\begin{tabular}{|c|c|c|c|c|c|}
\hline $\begin{array}{l}\text { Isolate } \\
\text { no. }\end{array}$ & Species & TSST & $\mathrm{SE}$ & Slime & Resistance \\
\hline 52 & S. aureus & - & $\mathrm{C}$ & ND & $\begin{array}{l}\text { Meth, Pen G, Tet, } \\
\text { Tmp, Fcd, Cd, Pi }\end{array}$ \\
\hline 137 & S. aureus & + & B & ND & $\mathrm{Km}, \mathrm{Cd}, \mathrm{Hg}$ \\
\hline 149 & S. aureus & + & $\mathrm{C}$ & ND & Pen $\mathrm{G}, \mathrm{Cd}$ \\
\hline 150 & S. aureus & + & $\mathrm{C}$ & $\mathrm{ND}$ & Susceptible \\
\hline 19 & S. epidermidis & - & B & - & $\mathrm{Cd}$ \\
\hline 48 & S. haemolyticus & + & - & + & Meth, PenG, \\
\hline 130 & S. haemolyticus & - & B & - & Susceptible \\
\hline 21 & S. hominis & + & - & + & Susceptible \\
\hline $29^{*}$ & S. hominis & - & - & + & Ery, Cd \\
\hline 47 & S. hominis & + & - & + & $\mathrm{Cd}$ \\
\hline 50 & S. hominis & - & $\mathrm{C}$ & + & $\mathrm{Tmp}$ \\
\hline 66 & S. hominis & + & - & - & Susceptible \\
\hline $86^{*}$ & S. hominis & - & A,B & - & Tet, Cd \\
\hline 89 & S. hominis & + & - & - & Tet, Cd \\
\hline 129 & S. hominis & - & B & - & Susceptible \\
\hline 133 & S. hominis & - & B & - & Tet \\
\hline 139 & S. saprophyticus & + & B & - & Susceptible \\
\hline 90 & S. schleiferi & - & $\mathrm{A}, \mathrm{B}$ & + & Susceptible \\
\hline 101 & S. schleiferi & - & A,B & - & Tet \\
\hline 108 & S. xylosus & + & - & + & Fcd, $\mathrm{Cd}, \mathrm{Hg}, \mathrm{Pi}$ \\
\hline 110 & S. xylosus & + & - & + & $\mathrm{Cd}, \mathrm{Hg}, \mathrm{Pi}$ \\
\hline $69^{*}$ & S. warneri & + & - & - & Susceptible \\
\hline 136 & S. warneri & - & $\mathrm{B}$ & - & Susceptible \\
\hline 138 & S. warneri & + & B & - & Susceptible \\
\hline Total & 24 & 13 & 15 & 8 & 14 \\
\hline
\end{tabular}

+, produced; -, not produced; Cd, cadmium; Ery, erythromycin; Fcd, fusidic acid; Hg, mercuric chloride; Km, kanamycin; PenG, penicillin G; Pi, propamidine isethionate; Meth, methicillin; Tet, tetracycline; Tmp, trimethoprim; ND, not determined.

* Nasal isolates. 
$39.3 \%$ and $S$. aureus $27.6 \%$ of the nasal flora of food handlers [25]. This may reflect differences in the distribution of CNS flora on different body sites [2] and in different populations living in different geographical regions.

The species distribution of CNS on the hands of the food handlers in this study showed a higher incidence of $S$. hominis $(23.6 \%)$ and $S$. warneri $(20.6 \%)$ than $S$. epidermidis $(3.4 \%)$. These results are similar to those reported by Larson and Burke [13], who isolated $S$. hominis more frequently from the skin flora of healthy adults and $S$. haemolyticus more frequently among hospitalised patients, but differ from the distribution of CNS on the skin of hospitalised patients where $S$. epidermidis is the most common species [30-35]. As the restaurant workers performed different duties relating to cooking and serving of different types of food, it is possible for some of them to have acquired staphylococcal flora from some of the food in addition to their own flora, because CNS have been isolated from different foodstuffs [16-21, 23, 24]. However, it is not known if any of the foodstuffs contributed to the CNS flora detected in this study.

The present study found that $8 \%$ of the CNS (including a nasal isolate of $S$. hominis) and $12.5 \%$ of $S$. aureus from the hands of the food handlers produced one or a combination of staphylococcal enterotoxins (Table 1). Despite the fact that only a small number of the CNS produced SE, their detection is significant because it confirms that $\mathrm{CNS}$ from different sources can produce SE [20]. It is also important because SE has not been studied extensively in CNS from food handlers. Whereas 10 of the 11 SE-producing CNS produced SEB, three of the four $S$. aureus isolates produced SEC. In a previous study, the majority of nasal $S$. aureus isolates from food handlers in Kuwait City produced either SEA or SEB [27]. The finding that $S$. aureus isolates from the hands produced mostly SEC would indicate that they did not originate from the workers' nares.

With the exception of tetracycline resistance, the incidence of antibiotic resistance was lower than that obtained from the skin flora of hospitalised patients [13,30-35]. However, detection of resistance to propamidine isethionate in $11.2 \%$ of the isolates was significant. Propamidine isethionate and quaternary ammonium compounds are components of household disinfectants and antiseptics [36,37], and resistance to propamidine isethionate is usually linked to resistance to quaternary ammonium compounds $[36,37]$. Therefore their resistance may diminish the usefulness of antiseptics containing them as hand-washing agents for removing enterotoxigenic staphylococci from the hands and preventing their cross-contamination.

No correlation was observed between slime production and antibiotic multi-resistance or between slime pro- duction and toxin production. Similarly, Alexander and Rimland [38] could not establish a correlation between slime production and antibiotic resistance or between slime production and pathogenicity in peritonitis caused by CNS, although slime production has been shown to correlate with antibiotic multi-resistance [3033 and with pathogenicity $[34,39]$ in CNS from hospital patients.

The detection of SE in CNS colonising the hands of these restaurant workers raises the important question as to whether these SEs can cause food poisoning. Although SEs have been detected previously from food incriminated in food poisoning [16, 20,21], from milk [22] and from the noses of food handlers [25], they have not been directly linked with food poisoning. However, as indicated previously, any staphylococcal strain producing SE can be involved in food poisoning [29]. In addition, the present study has demonstrated that enterotoxigenic CNS can co-exist with enterotoxigenic $S$. aureus, colonising both nasal and hand surfaces. Therefore, it is possible for enterotoxigenic CNS to contribute to food poisoning given the right conditions. It is possible that they have been involved in food poisoning in the past in association with $S$. aureus, but have been overlooked once enterotoxigenic $S$. aureus was isolated. As cautioned by Crass and Bergdoll [16], CNS should not be ignored but should be considered with $S$. aureus in suspected cases of staphylococcal food poisoning. Also, increased awareness of enterotoxigenic CNS carriage among food handlers is necessary to avoid contamination of food with these strains.

This project was supported in part by Kuwait University Research Administration Grant no. MI 091. We thank Rhoñe-Poulenc Rorer, UK for the gift of propamidine isethionate.

\section{References}

1. Baird-Parker AC. The staphylococci: an introduction. Soc Appl Bacteriol Symp Ser 1990; 19: 1S-8S.

2. Marples RR, Richardson JF, Newton FE. Staphylococci as part of the normal flora of human skin. Soc Appl Bacteriol Symp Ser 1990; 19: 93S-99S

3. Noble WC. Systematics and the natural history of staphylococci. 2. Soc Appl Bacteriol Symp Ser 1990; 19: 39S-8S.

4. Bergdoll MS. Staphylococcal intoxications In: Rieman $M$, Bryan FL (eds) Foodborne infections and intoxications, 2nd edn. New York, NY, Academic Press. 1979: 463-523.

5. Dick MIB, Grant AJ, Linford GJ. Staphylococcal enterotoxins. In: Buckle KA, Davey JA, Eyles MJ, Hocking AD, Newton KG, Stuttard EJ (eds) Foodborne microorganisms of Public Health significance. Sydney, J M Executive Printing Service 1993: $271-284$.

6. Arbuthnott JP, Coleman DC, de Azavedo JS. Staphylococcal toxins in human disease. Soc Appl Bacteriol Symp Ser 1990: 19: 101S-107S.

7. Tranter HS, Brehm RD. The detection and aetiological significance of staphylococcal enterotoxins. Rev Med Microbiol 1994; 5: 56-64.

8. Wieneke AA, Roberts D, Gilbert RJ. Staphylococcal food poisoning in the United Kingdom, 1969-90. Epidemiol Infect 1993; 110: 519-531.

9. Karchmer AW, Archer GL, Dismukes WE. Staphylococcus epidermidis causing prosthetic valve endocarditis: microbiolo- 
gic and clinical observations as guide to therapy. Ann Intern Med 1983; 98: 447-455.

10. Christensen GD, Bisno AL, Parisi JT, McLaughlin B, Hester MG, Luther RW. Nosocomial septicemia due to multiple antibiotic-resistant Staphylococcus epidermidis. Ann Intern Med 1982; 96: $1-10$.

11. Goldmann DA, Pier GB. Pathogenesis of infections related to intravascular catheterization. Clin Microbiol Rev 1993; 6: 176-192.

12. Beard-Pegler MA, Gabelish CL, Stubbs E et al. Prevalence of peritonitis-associated coagulase-negative staphylococci on the skin of continuous ambulatory peritoneal dialysis patients. Epidemiol Infect 1989; 102: 365-378.

13. Larson RA, Burke JP. The epidemiology and risk factors for nosocomial catheter-associated bacteriuria caused by coagulasenegative staphylococci. Infect Control 1986; 7: 212-215.

14. Kreiswirth BN, Schlievert PM, Novick RP. Evaluation of coagulase-negative staphylococci for ability to produce toxic shock syndrome toxin-1. $J$ Clin Microbiol 1987; 25: 2028-2029.

15. Kahler RC, Boyce JM, Bergdoll MS, Lockwood WR, Taylor MR. Toxic shock syndrome associated with TSST-1 producing coagulase-negative staphylococci. Am J Med Sci 1986; 292: $310-312$.

16. Crass BA, Bergdoll MS. Involvement of coagulase-negative staphylococci in toxic shock syndrome. J Clin Microbiol 1986 23: 43-45.

17. Scheifele DW, Bjornson GL. Delta toxin activity in coagulasenegative staphylococci from the bowel of neonates. $J$ Clin Microbiol 1988; 26: 279-282.

18. Butt HL, Dunstan RH, McGregor NR, Roberts TK, Zerbes M, Klinberg IJ. An association of membrane-damaging toxins from coagulase-negative staphylococci and chronic orofacial muscle pain. J Med Microbiol 1998; 47: 577-584.

19. Rosec JP, Guiraud JP, Dalet C, Richard N. Enterotoxin production by staphylococci isolated from food in France. Int $J$ Food Microbiol 1997; 35: 213-221.

20. Hoover DG, Tatini SR, Maltais JB. Characterization of staphylococci. Appl Environ Microbiol 1983; 46: 649-660.

21. Lotter LP, Genigeorgis LA. Deoxyribonucleic acid base composition and biochemical properties of certain coagulasenegative cocci. Appl Microbiol 1975; 29: 152-158.

22. Vernozy-Rozand C, Mazuy C, Prevost G et al. Enterotoxin production by coagulase-negative staphylococci isolated from goats' milk and cheese. Int J Food Microbiol 1996; 30: $271-280$.

23. Orden JA, Goyache J, Hernandez J, Domenenech A, Suarez G, Gomez-Lucia E. Production of staphylococcal enterotoxins and TSST-1 by coagulase-negative staphylococci isolated from ruminant mastitis. Zentrabl Veterinarmed [B] 1992; 39: 144-148.

24. Adesiyun AA, Usman B. Isolation of enterotoxigenic strains of staphylococci from dogs. Vet Microbiol 1983; 8: 459-468.

25. Francisco Polledo JJ, Garcia ML, Moreno B, Menes I. Importance of food handlers as a source of enterotoxigenic staphylococci. Zentrabl Bacteriol Mikrobiol Hyg [B] 1985; 181: 364-367.

26. Soares MJS, Tokumaru-Miyazaki NH, Noleto ALS, Figueiredo AMS. Enterotoxin production of Staphylococcus aureus and detection of Brazilian epidemic MRSA clone (III::B:A) among isolates from food handlers. $J$ Med Microbiol 1997; 46: 214-221.

27. Al-Bustan MA, Udo EE, Chugh TD. Nasal carriage of enterotoxin-producing Staphylococcus aureus among restaurant workers in Kuwait City. Epidemiol Infect 1996; 116: 319-322.

28. Soto A, Saldias ME, Oviedo P, Fernandez M. [Prevalence of Staphylococcus aureus among food handlers from a metropolitan university in Chile.] Rev Med Chil 1996; 124: $1142-1146$.

29. Bergdoll MS. Importance of staphylococci that produce nanogram quantities of enterotoxin. Int $J$ Med Microbiol Virol Parasitol Infect Dis 1995; 282: 1-6.

30. Udo EE, Jacob LE, Chugh TD. Antimicrobial resistance of coagulase-negative staphylococci from a Kuwait hospital. Microbial Drug Resist 1995; 1: 315-320.

31. Jones JW, Scott RJD, Morgan J, Pether JVS. A study of coagulase-negative staphylococci with reference to slime production, adherence, antibiotic resistance patterns and clinical significance. $J$ Hosp Infect 1992; 22: 217-227.

32. Deighton MA, Franklin JC, Spicer WJ, Balkau B. Species identification, antibiotic sensitivity and slime production of coagulase-negative staphylococci isolated from clinical specimens. Epidemiol Infect 1988; 101: 99-113.

33. Kleeman KT, Bannerman TL, Kloos WE. Species distribution of coagulase-negative staphylococcal isolates at a community hospital and implications for selection of staphylococcal identification procedures. $J$ Clin Microbiol 1993; 31: $1318-1321$.

34. Ishak MA, Gröschel DHM, Mandell GL, Wenzel RP. Association of slime with pathogenicity of coagulase-negative staphylococci causing nosocomial septicemia. J Clin Microbiol 1985; 22: 1025-1029.

35. Kloos WE, Bannerman TL. Update on clinical significance of coagulase-negative staphylococci. Clin Microbiol Rev 1994; 7: $117-140$.

36. Emslie KR, Townsend DE, Grubb WB. A resistance determinant to nucleic acid-binding compounds in methicillin-resistant Staphylococcus aureus. J Med Microbiol 1985; 20: 139-145.

37. Leelaporn A, Paulsen IT, Tennent JM, LittleJohn TG, Skurray RA. Multidrug resistance to antiseptics and disinfectants in coagulase-negative staphylococci. J Med Microbiol 1994; 40: $214-220$.

38. Alexander W, Rimland D. Lack of correlation of slime production with pathogenicity in continuous ambulatory peritoneal dialysis peritonitis caused by coagulase-negative staphylococci. Diagn Microbiol Infect Dis 1987; 8: 215-220.

39. Christensen GD, Simpson AW, Bisno AL, Beachy EH Adherence of slime-producing strains of Staphylococcus epidermidis to smooth surfaces. Infect Immun 1982; 37: $318-326$. 